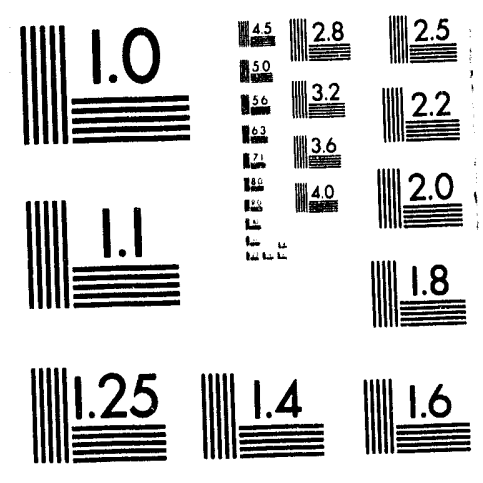



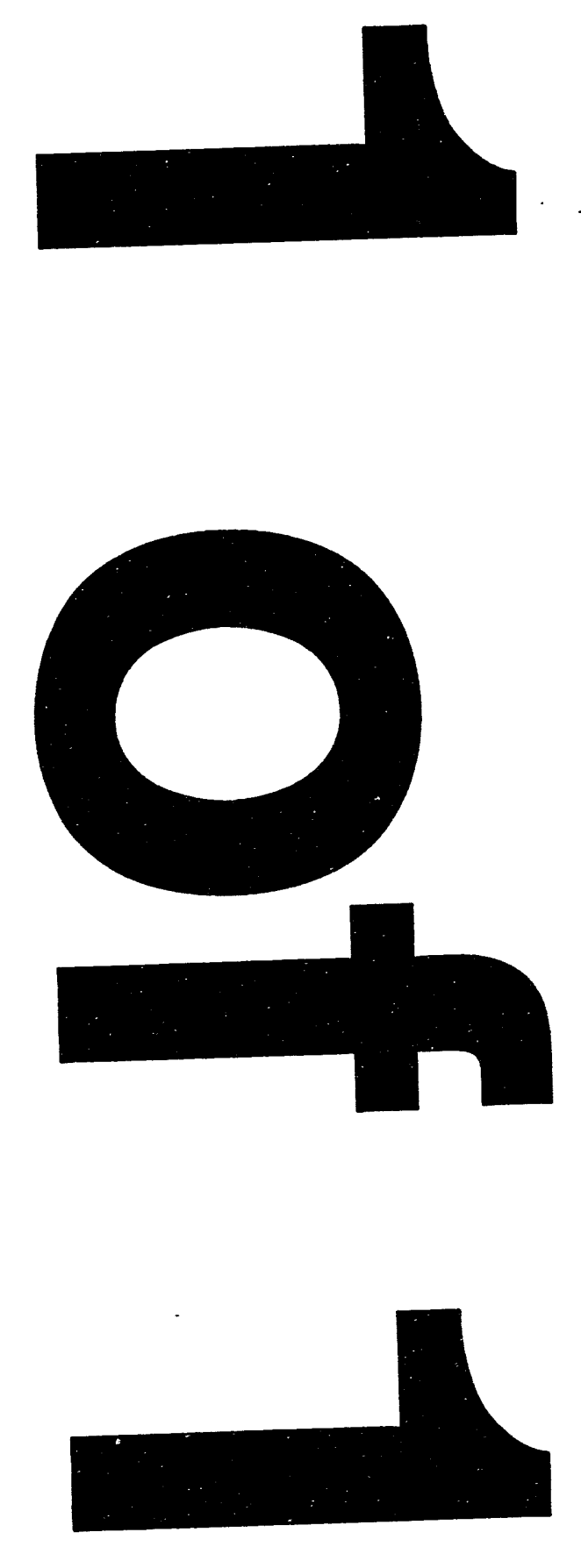
Title:

Decoherence, Chaos, The Quantum and the Classical

Author(s):

W. H. Zurek and J.P. Paz

Submitted to:

Symposium on the Foundations of Modern Physics 199:

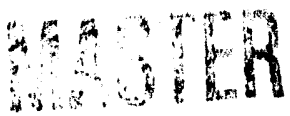

\section{Los Alamos} WATIONAL LABOAATOOY

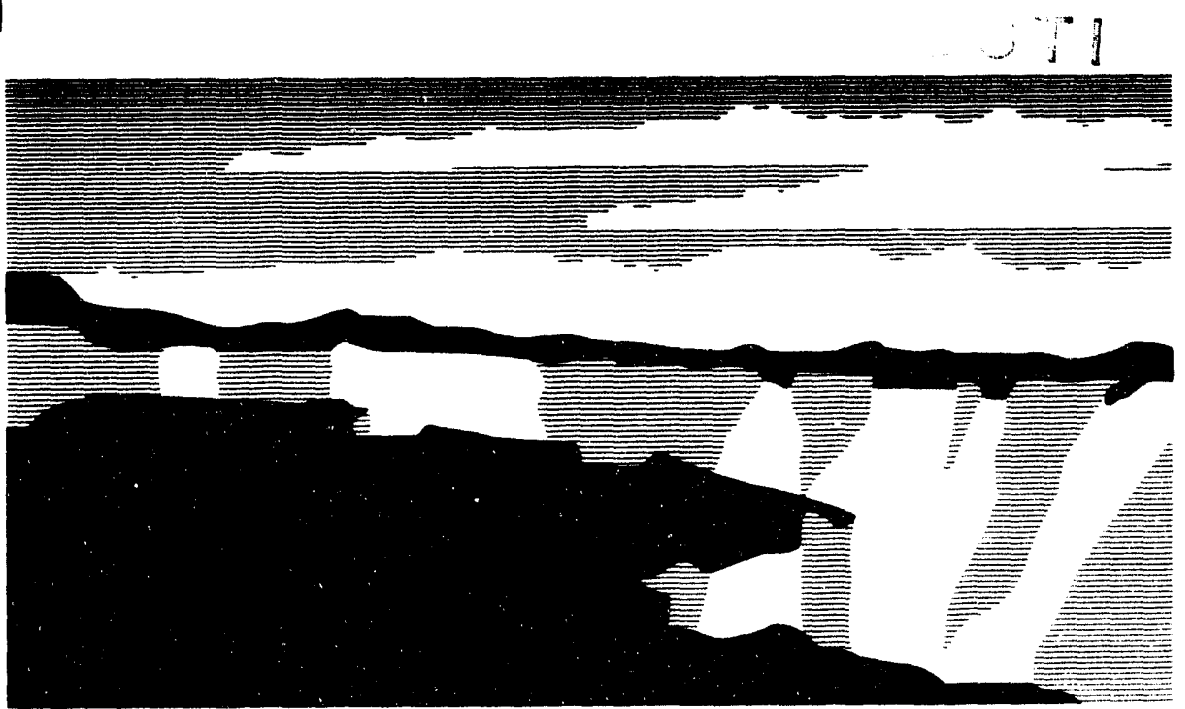

Los Alamos National Laboratory, an affirmative action/equal opoontunity emplofyer, is operated by the University of California for the U.S. Department of Energy under contract W-7405-ENG-36. By acceptance of this anticle, the publisher recognizes that the U.S. Government retains a nonexclusive, royalty-free license to publish or reproduce the published form of this contribution, or to allow others to do so, for U.S. Government purposes. The Los Alamos National Laboratory requests that the publisher identify this article as work performed under the auspices of the U.S. Department of Energy. 


\title{
DECOHERENCE, CHAOS, THE QUANTUM AND THE CLASSICAL
}

\author{
W. H. Zurek and J. P. Paz \\ Theoretical Astrophysics, T-6, MS B288 \\ Los Alamos National Laboratory, Los Alamos, NM 87545
}

\begin{abstract}
The key ideas of the environment-induced decoherence approach are reviewed. Application of decoherence to the transition from quantum to classical in open quantum systems with chaotic classical analogs is described. The arrow of time is, in this context, a result of the information loss to the correlations with the environment. The asymptotic rate of entropy production (which is reached quickly, on the dynamical timescale) is independent of the details of the coupling of the quantum system to the environment, and is set by the Lyapunov exponents. We also briefly outline the existential interpretation of quantum mechanics, justifying the slogan "No information without representation".
\end{abstract}

\section{Introduction}

Quantum theory allows many more states for the objects described by it than we seem to encounter. Moreover, quantum dynamics (especially the dynamics required to model measurements, but also dynamics of quantum systems whose classical analogs exhibit "chaos") takes simple, localized initial states of individual systems into complicated nonlocal superpositions. We do not perceive such superpositions. Macroscopic objects always appear to us in a small classical subset of a much larger quantum menu which is in principle available in the Hilbert space. No one has made this point more clear than Albert Einstein, who, in a 1954 letter to Max Born writes": "Let $\Psi_{1}$ and $\Psi_{2}$ be solutions of the same Schrödinger equation ... When the system is macroscopic and $\Psi_{1}$ and $\Psi_{2}$ are 'narrow' with respect to the macrocoordinates, then ... [typically] this is no longer the case for $\Psi=$ $\Psi_{1}+\Psi_{2}$. Narrowness with respect to macrocoordinates is not only independent of the principles of quantum mechanics, but, moreover, incompatible with them..." Hence, predictions of quantum theory seem to be in conflict with our perceptions.

The purpose of the decoherence approach is to show that this conflict is only apparent: Classical behavior of systems we encounter as well as the uniqueness of our perceptions can be accounted for by the "openness" of the macroscopic objects, including the devices employed by us, the observers, to keep records. The purpose of this paper is twofold:

i) We will outline key ingredients of the decoherence approach to the transition from quantum to classical, including the recent developments, such as the predictability sieve and the existential interpretation (a more complete summary is available in the literature ${ }^{2,3,4}$ ). 
ii) We will briefly discuss the transition from quantum to classical in open quantum systems which are classically chaotic (a more complete presentation will be published elsewhere ${ }^{5}$ ).

\section{Decoherence}

The key point of the decoherence approach is simple: There is a basic difference between the predictions of quantum theory for quantum systems which are closed (isolated) and open (interacting with their environments). In the case of a closed system, Schrödinger equation and the superposition principle apply literally. By contrast, for an open quantum system superposition principle is not valid. There the relevant physics is quite different, as was realized already some time ago in the context of condensed matter physics, quantum chemistry, etc. However, the paramount implications of the interaction with the environment for the transition from quantum to classical were not appreciated until recently. Especially important is the fact that the evolution of open quantum systems violates the "equal rights amendment" pointed out by Einstein in his comment above, and guaranteed for each and every state in the Hilbert space of a closed system by the superposition principle. Thus, decoherence results in a negative selection process which dynamically eliminates non-classical states.

The distinguishing feature of classical observables, the essence of "classical reality" is the persistence of their properties - the ability of systems to exist in predictably evolving states, to follow a trajectory which may be chaotic, but is deterministic. This suggests the relative stability - or, more generally, predictability of the evolution of quantum states - as a criterion which decides whether they will be repeatedly encountered by us, the observers, and used as the ingredients of the "classical reality". The characteristic feature of the decoherence process is that a generic initial state - a random sample taken from the vast menu present in the Hilbert space where every state is a possibility allowed by quantum mechanics will be dramatically altered on a characteristic decoherence timescale: Only certain stable states (which turn out to be, in a sense, decay products of the other states) will be left on the scene. Another key idea which must be kept in mind is that the states of our records must be also treated as a part of the sample. And only stable records - states of neurons or other memory devices which can survive decoherence and maintain correlations with the measured system - can be used as a physical basis for the perception of classical reality.

Quantum measurement is a classic example of a situation in which a coupling of a macroscopic quantum apparatus $\mathcal{A}$ and a microscopic measured system $\mathcal{S}$ forces the composite object into a correlated but usually exceedingly unstable state: In a notation where $\left|A_{0}\right\rangle$ is the initial state of the apparatus and $|\psi\rangle$ the initial state of the system, evolution establishing an $\mathcal{A}-\mathcal{S}$ correlation is described by:

$$
|\psi>| A_{o}>=\sum_{k} a_{k}\left|\sigma_{k}>\right| A_{o}>\rightarrow \sum_{k} a_{k}\left|\sigma_{k}>\right| A_{k}>=\mid \Phi>.
$$


A popular example is the Stern-Gerlach apparatus. There the states $\left|\sigma_{k}\right\rangle$ describe orientations of the spin and the states $\left|A_{k}\right\rangle$ are the spatial wavefunctions centered on the trajectories corresponding to different eigenstates of the spin. When the separation of the beams is large, the overlap between them tends to zero ( $<A_{k} \mid A_{k}^{\prime}>\sim \delta_{k k^{\prime}}$ ). This is a precondition for a good measurement. Moreover, when the apparatus is not consulted, $\mathcal{A}-\mathcal{S}$ correlation would lead to a mixed density matrix for the system $\mathcal{S}$ :

$$
\rho_{\mathcal{S}}=\sum_{k}\left|a_{k}\right|^{2}\left|\sigma_{k}><\sigma_{k}\right|=\operatorname{Tr}_{\mathcal{A}}|\Phi><\Phi| .
$$

However, this single, instantaneous pre-measurement quantum correlation does not provide a sufficient foundation to build a correspondence between quantum formalism and the familiar classical reality. It only allows for non-separable, Einstein Podolsky - Rosen quantum correlations between $\mathcal{A}$ and $\mathcal{S}$, for pairing of an arbitrary state - including non-local, non-classical superpositions of localized states of the apparatus (observer) - with the corresponding relative state of the other system. This is a prescription for a Schrödinger cat, not a resolution of the measurement problem. What is needed is a fixed set of states in which classical systems can safely exist, but which instantly decay when superposed. What is needed is a an effective superselection rule which will "outlaw" superpositions of these preferred pointer states. It cannot be absolute - there must be a timescale sufficiently short, or interaction strong enough to render it invalid, for otherwise measurements could not be performed at all. It should become more effective with the increase in size of the system. It should follow from quantum mechanics, superposition principle notwithstanding. It should apply in general, to all objects (not just idealized models of a quantum apparatus) and allow one to deduce elements of the familiar reality of everyday experience - including the spatial localization of macroscopic systems - from hamiltonians and not much else.

Environment - induced decoherence fits this bill. The transition from a pure state $|\Phi\rangle\langle\Phi|$ to the effectively mixed $\rho_{\mathcal{A S}}$ can be accomplished by coupling the apparatus $\mathcal{A}$ to the environment $\mathcal{E}$. The requirement for getting rid of the unwanted, excessive, EPR-like correlations, Eq. (1), boils down to the demand that the correlations between the record-keeping pointer states of the apparatus and the measured system ought to be preserved in spite of an incessant measurement-like interaction between the apparatus pointer and the environment. In simple models of the apparatus this can be assured by postulating existence of a pointer observable with eigenstates (or, more precisely, eigenspaces) which remain unperturbed in course of evolution of the open system: This "nondemolition" requirement will be exactly satisfied when the pointer observable $\Lambda$ commutes with the total hamiltonian generating the evolution of the system:

$$
\left[H+H_{\text {int }}, \Lambda\right]=0 \text {. }
$$

For an idealized quantum apparatus this condition can be assumed, and - providing that an apparatus is in one of the eigenstates of $\Lambda$ - it leads to an uneventful 
evolution:

$$
\left|A_{k}>\right| \mathcal{E}_{0}>\rightarrow\left|A_{k}>\right| \mathcal{E}_{k}(t)>
$$

However, when the initial state is a superposition corresponding to different eigenstates of $\Lambda$, the environment will evolve into an $\left|A_{k}\right\rangle$ - dependent state:

$$
\left(\sum_{k} a_{k} \mid A_{k}>\right)\left|\mathcal{E}_{0}>\rightarrow \sum_{k} a_{k}\right| A_{k}>\mid \mathcal{E}_{k}(t)>\text {. }
$$

The decay of the interference terms is inevitable. The environment causes decoherence only when the apparatus is forced into a superposition of states which are distinguished by their effect on the environment. The resulting continuous destruction of the interference between the eigenstates of $\Lambda$ leads to an effective environmentinduced superselection. Only states which are stable in spite of decoherence can exist long enough to be accessed by the observers - to be counted as the elements of the familiar, reliably existing reality.

Effective reduction of the state vector immediately follows. When the environment becomes correlated with the apparatus:

$$
|\Phi>| \mathcal{E}_{0}>\rightarrow \sum_{k} a_{k}\left|A_{k}>\right| \sigma_{k}>\left|\mathcal{E}_{k}(t)>=\right| \Psi>
$$

but is not consulted (so that it must be traced out) then we will have

$$
\rho_{\mathcal{A S}}=\operatorname{Tr}_{\tilde{\varepsilon}}|\Psi><\Psi|=\sum_{k}\left|a_{k}\right|^{2}\left|A_{k}><A_{k}\right|\left|\sigma_{k}><\sigma_{k}\right|
$$

Only correlations between the pointer states and the corresponding relative states of the system retain their predictive validity. This form of $\rho_{\mathcal{A S}}$ follows providing that the environment becomes correlated with the set of states $\left\{\mid A_{k}>\right\}$ (it could have been any other set) and that it has acted as a good measuring apparatus, so that $\left\langle\mathcal{E}_{k}(t) \mid \mathcal{E}_{k^{\prime}}(t)\right\rangle=\delta_{k k^{\prime}}$ - the states of the environment correlated with the different outcomes are orthogonal.

\section{Reduction of the Wavepacket in quantum Brownian motion}

\subsection{Predictability Sieve}

Can a similar process be responsible for the classical behavior of systems which cannot be idealized as simply as an abstract apparatus? The crucial difference arises from the fact that, in general, there will be no (non-trivial) observable which will commute with both parts of the total hamiltonian $H+H_{\text {int }}$. Thus all of the states - and all of the correlations - will evolve on some timescale. The distinction between various states will now have to be quantitative, rather than qualitative: The majority of states will deteriorate on the decoherence timescale. This is the time required for the reduction of the wavepacket. For non-classical states of macroscopic objects, it is many orders of magnitude shorter than the dynamical timescale - so 
short that, from the point of view of the observers responding on their dynamical timescale, it can be regarded as instantaneous.

The interaction with the environment will continue to play a crucial role: Monitoring of the to - be - classical observable by the environment is still the process responsible for decoherence, and $H_{\text {int }}$ determines the set of states which leave distinguishable imprints in the environment. For example, the commutation condition, Eq. (3), for the interaction hamiltonian alone explains the approximate localization of classical states of macroscopic objects: The environment is coupled nearly always through the coordinate $x$ (interactions depend on distance) and, therefore, states which are localized will be favored ${ }^{6}$ : This feature of the preferred states follows from the form of the interaction alone - it does need to be put in by hand.

However, this cannot be the whole story. The kinetic term in the hamiltonian $\left(p^{2} / 2 m\right)$ does not commute with the position observable. Therefore, exact position eigenstates - which have to be, because of the Heisenberg indeterminacy, completely "nonlocal in momentum" - are also unstable. We need a more systematic procedure to filter out non-classical states. A natural generalization of absolutely stable pointer states of the apparatus are the most predictable states of less idealized open quantum systems. An algorithm for "trying out" all of the states in the Hilbert space can be readily outlined ${ }^{3}$ : For each candidate initial state we can calculate the density matrix which obtains from its evolution in contact with the environment, compute its entropy as a function of time, and - at some instant much in excess of a typical decoherence timescale - construct a list of the pure initial states ordered according to how much entropy was generated, or how much predictability was lost in the process. The most predictable states near the top of the list would be, in effect, most classical. A similar procedure could be also used to compare the predictability of mixed initial states.

This predictability sieve was recently proposed ${ }^{3}$ and implemented for a harmonic oscillator with the resulting evolution of the reduced density matrix generated by the appropriate master equation ${ }^{7}$. For a weakly damped harmonic oscillator, pure states selected by the predictability sieve turn out to be the familiar coherent states ${ }^{8}$. This is true in spite of the fact that the stability of the states is crucially influenced by their dispersion in position. For instance, for pure states the rate of purity loss (quantified by the linear entropy given by $\operatorname{Tr} \rho^{2}$ ) is proportional to their dispersion in $x$;

$$
\frac{d}{d t} \operatorname{Tr} \rho^{2} \sim\left(<\psi\left|x^{2}\right| \psi>-|<\psi| x|\psi>|^{2}\right) \text {. }
$$

Most predictable mixtures turn out to be also gaussian and - for a weakly damped oscillator - symmetric in $x$ and $p$.

\subsection{Towards "Classical Reality"}

It is clear from this recapitulation of the decoherence process and its consequences that naive attempts at an interpretation based solely on the instantaneous eigenstates of the density matrix of a single system are, at best, a poor caricature 
of the implications of environment - induced decoherence. This oversimplification (which ignores the original focus on correlations ${ }^{9}$, so essential in the discussion of the information acquisition through measurements, the existence of the preferred sets of states, or the issue of predictability and determinism, crucial in the definition of effective classicality) may be, of course, easier to describe. We shall discuss an existential interpretation which focuses primarily on the stable existence (or predictable evolution) of states as a defining feature of "classical reality." First, however, let us address several specific comments which are sometimes brought up by the critics of the decoherence approach.

For example, it is often emphasized that the concept of systems is crucial in the discussion of decoherence ${ }^{8}$. Is this "artificial division of the physical world" a reason for dismissing decoherence as a step towards a resolution of the measurement problem? Certainly not! The problem of measurement cannot be even stated without dividing the Universe into a system and the apparatus ${ }^{3}$. In absence of such a division any closed system will evolve in a completely deterministic, unitary manner, in accord with the Schrödinger equation. Difficulties with interpretation start only when one realizes that such a deterministic trajectory in the Hilbert space takes a composite object (i.e. apparatus/observer plus the measured system) from an initial state in which each of them has a definite property to a state where neither of them appears to be entitled to "a state of its own".

Since the problem of measurement cannot be posed without recognizing that systems exist, there is no need to apologize for assuming their existence in search for its resolution: And the addition of the environment is not an addition of an arbitrary extra ingredient, but a recognition of an existing component which makes idealized models of measurements more realistic. Indeed, in all familiar situations observations are carried out by "bleeding off" a fraction of the information already imprinted on and present in the environment (for example, in the photons scattered by the object we see). If the minuscule fraction of the record imprinted in just a few select kinds of environment that we are capable of deciphering suffices to satisfy our information - gathering needs, it is easy to appreciate the accuracy with which all of the environmental degrees of freedom are monitoring observables of macroscopic objects. This is not to say that the issue of the definition of the system can be dismissed altogether or considered unimportant: On the contrary, the role systems play both in the formulation of the measurement problem and in its resolution deserves attention.

Details of the definition of a system will not alter the resolution of the measurement problem suggested by decoherence: The exact location of the boundary between the system and the environment matters very little for the onset of environment-induced superselection. The correlations with the macroscopically distinguishable states of the system spread through the environment very quickly, and a single unaccounted for correlation suffices to destroy quantum coherence.

The next issue often raised by the critics is the applicability of the decoherence approach to the Universe as a whole and its relation to the consistent histories 
approach $^{10}$. The Universe is a closed system, so it does not have an environment. However, macroscopic subsystems within it (including recording apparatus and observers) do have environments. Hence, the decoherence program can be readily implemented in this setting. The projection operators which define sequences of events in the consistent histories approach would then have to satisfy not just the probability sum rules. (The resulting consistency conditions turn out to be easy to satisfy exactly by numerous sets of projection operators which have nothing to do with the "familiar reality.") Rather, the process of decoherence singles out events and observables which become (relatively permanently) "recorded" as a result of environmental monitoring. For example, when a well-defined pointer basis exists, the histories consisting of sequences of pointer states are consistent: Approximate consistency of the familiar classical histories as a consequence of environment - induced superselection ${ }^{3,11}$. Thus the additivity of probabilities of histories expressed in terms of the "usual" observables appears to be guaranteed by the efficiency with which unstable states and the corresponding off-diagonal terms of the density matrix in the preferred pointer basis representation are removed by a coupling with the environment.

\section{Decoherence, Chaos and the Second Law}

According to the picture presented above, an open quantum system prepared in a generic initial state would suffer a very fast decoherence process after which its state will become an approximate mixture of pointer states (e. g., coherent states in the case of an underdamped particle $\left.{ }^{8}\right)$. In this context, it is natural to ask what other consequences the openness of the quantum system has. One should first notice an important fact: the effectiveness of decoherence does not necessarily imply noticeable presence of other observable dissipative effects. In fact, as has been pointed out before, in a typical quantum Brownian systems one has;

$$
\tau_{d e c}=\gamma^{-1}\left(\frac{\lambda_{d B}}{\Delta x}\right)^{2}
$$

where $\gamma$ is the relaxation rate, $\lambda_{d B}$ is the thermal de Broglie wavelength and $\Delta x$ is the distance over which the quantum state is coherent. This implies that in realistic situations the decoherence timescale can be many orders of magnitude smaller than the characteristic relaxation timescale: For typical macroscopic parameters $\tau_{\text {dec }}$ turns out to be 40 orders of magnitude smaller than $1 / \gamma^{2}$

Thus, the transfer of energy to the reservoir can be made arbitrarily slow, but what about the entropy? Here we will briefly analyze the rate at which the information initially contained in the system is lost in the correlations with the environment and point out a major difference between quantum analogs of systems which are classically chaotic or regular. The main result (details of which will be published elsewhere ${ }^{5}$ ) is that for quantum open systems whose classical analogs are chaotic, the rate of von Neumann entropy production reaches, on a dynamical timescale, an asymptotic value whihc is independent of the coupling with the environment and 
is dominated by the largest positive Lyapunov exponent. On the way to this result we shall also point out that:

i) Decoherence limits, from below, the scale of the structure of quantum states in the phase space (i. e., Wigner function $W$ cannot vary on a scale set by a certain characteristic dispersion in momentum, $\sigma_{c}$ ).

ii) As a result of this limit on the "resolution" of $W$ for open chaotic quantum systems, classical dynamics will hold for periods much longer than for closed systems. The Poison bracket, which generates Liouville evolution, and the Moyal bracket, which generates quantum evolution in phase space, approximately coincide when evaluated for such "blurred" $W$ 's. Thus, decoherence resolves the longstanding question of the correspondence between quantum and classical in the context of chaotic systems.

To make our analysis concrete let us address first a simpler issue asking what are the properties of the quantum dynamics of a system which classically exhibits the defining property of chaos - exponential divergence of trajectories - and which is coupled to an environment. As a tractable model of such system we will discuss the inverted harmonic oscillator and represent the effects of the coupling with the environment through a master equation (using the high temperature Markovian approximation). In such a case, the Wigner function obeys Fokker-Planck equation ${ }^{7}$ :

$$
\dot{W}=-\omega\left(p \partial_{x}+x \partial_{p}\right) W+2 \gamma \partial_{p}(p W)+\frac{\omega}{2} \sigma_{c}^{2} \partial_{p p} W
$$

Above, $\omega$ is the frequency of the unstable oscillator (which also plays the role of the Lyapunov exponent), $\gamma$ is the relaxation rate and the coordinate $x$ has been rescaled $x \rightarrow m \omega x$ where $m$ is the mass. The characteristic (momentum) dispersion, which will play a crucial role in what follows, is defined as;

$$
\sigma_{c}^{2}=\frac{2 D}{\omega}
$$

where the diffusion coefficient is $D=2 \gamma m k_{B} T$ and $T$ is the temperature of the environment. The three terms on the right-hand side of Eq. (10) play different roles: The first term generates a Liouville flow in the phase space. (In the case of linear systems Poisson and Moyal brackets coincide.) The second one causes relaxation - gradual loss of energy to the reservoir. The third is a diffusion term which is responsible for decoherence ${ }^{2,12}$.

In general, three different stages of the evolution of $W$ can be identified. First, a generic initial state, containing interference effects - which correspond to a nonpositive, rapidly oscillating Wigner function - will decohere very fast. This will take place on a timescale (9), which is, for typical macroscopic parameters, much shorter than any dynamical timescale. To illustrate how the interference structure present in the Wigner function on scales of the order of $l_{p}$ (e. g., oscillations of such wavelength in the interference terms of $W$ ) will disappear on a timescale of the 
order $\tau_{d e c}=\omega^{-1}\left(l_{p} / \sigma_{c}\right)^{2}$, one can repeat calculations outlined elsewhere ${ }^{2.12}$. For example, when the initial state is a superposition of two coherent states separated by a distance $L, l_{p} \propto \hbar / L$, and the ratio between the wavelength of the "interference fringes" in momentum and $\sigma_{c}$ is $\sigma_{c}^{2} / l_{p}^{2}=\gamma / \omega\left(L / \lambda_{d B}\right)^{2}$. Therefore, the decoherence time is given by Eq. (9).

In addition to this very fast decoherence stage, the evolution has two qualitatively different stages - reversible and irreversible - whose origin can be understood as follows: For the unstable oscillator, as well as for a general chaotic system, there are two competing processes affecting the evolution, the Liouville flow and the diffusion. The instability present in the Hamiltonian tries to exponentially "expand" the wavepacket in one direction (the coordinate associated with this expanding direction is $v=p-x$ ) and "contract" it along another direction (the contracting coordinate is $u=p+x$ ). Exponential expansion and contraction produce squeezing of the Wigner function. Diffusion, which is the main ingredient of decoherence, opposes this exponential contraction, as it induces spreading of the wavepacket. (By contrast, it has little effect on the exponential expansion.) The result of this competition is a balance which implies the existence of a critical width for the Wigner function along the contracting direction. This is $\sigma_{c}$, which is given by (11). In fact, using the above equation one can show that an initial Wigner function (smooth on a scale of the order of $\sigma_{c}$ ) will evolve, after a few dynamical times $\omega^{-1}$ into:

$$
W(u, v, t) \approx \frac{1}{\sqrt{2 \pi \sigma_{c}^{2}}} e^{-u^{2} / 2 \sigma_{c}^{2}} e^{-\omega t} \int_{-\infty}^{\infty} d u W\left(u, v_{0}, t=0\right) .
$$
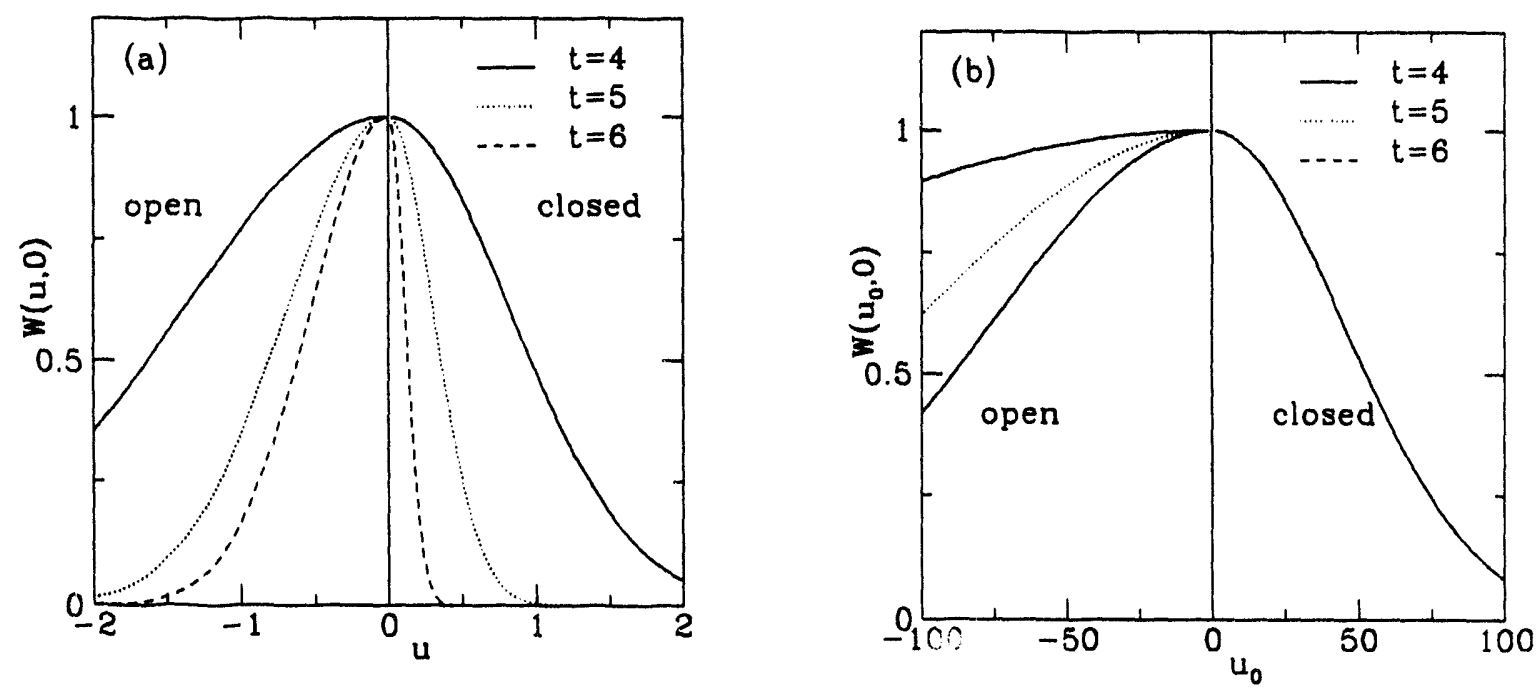

Figure 1: $W(u, v)$ is plotted (for fixed value of $v$ ) as a function of the contracting coordinate $u$. (a), and the "comoving" coordinate $u_{0},(\mathrm{~b})$. Plots in the left correspond to an open system evolving according to equation (10). The existence of a critical width, which fixes a lower bound for the small scale structure of $W$ is clearly seen. 
Therefore, when the initial state has a characteristic spread which is larger than $\sigma_{c}$, and no substructure on smaller scales, the initial stage of the evolution will be reversible and characterized by negligible entropy production: The state will contract in one direction and expand in the other with diffusion contributing little to its evolution for as long as the the smallest scale of variations of $W$ will be much larger than $\sigma_{c}$. The irreversible stage of the evolution begins when, due to the contraction, small scale structure shrinks to the characteristic size $\sigma_{c}$ (see Figure 1). When this happens the contraction stops but the expansion continues: Therefore, during the irreversible evolution, the area enclosed by the $1-\sigma$ contour of the Wigner function grows at a rate fixed by the expansion rate (which is equal to $\omega)$. This area can be related to the entropy (in effect, entropy is a natural logarithm of that volume in the phase space). Therefore, we conclude that in the third stage of the evolution, the entropy grows at a rate which is fixed by $\omega^{-1}$.

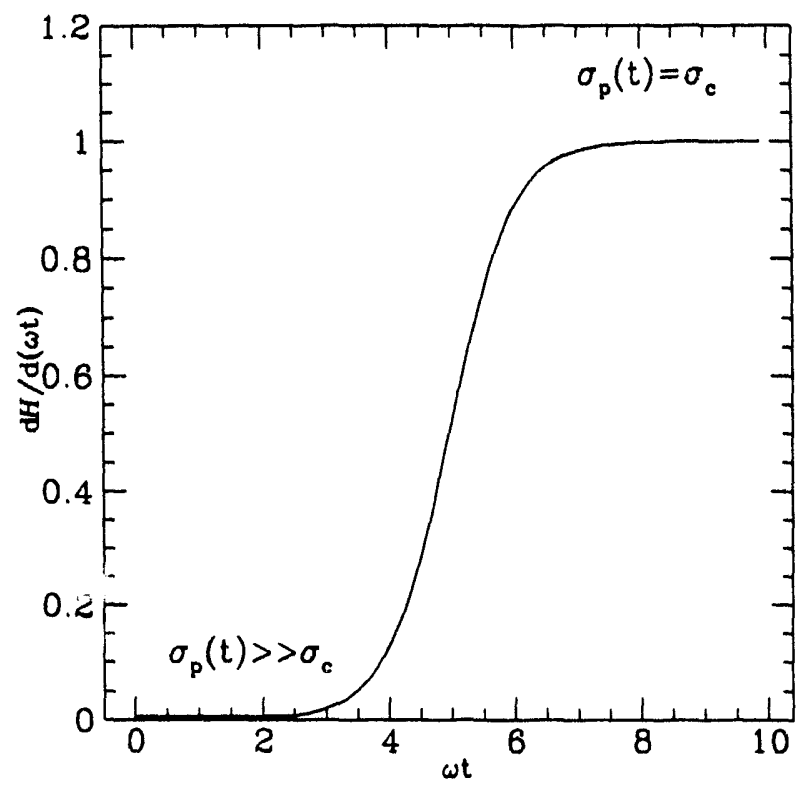

Figure 2: The von Neumann entropy production rate as a function of time for an unstable harmonic oscillator considered here. The wavepacket evolves according to equation (10) and the reversible and irreversible phases are clearly distinguished by the rate of entropy production.

A clear distinction between the last two stages of the evolution is apparent in Figure 2 where the von Neumann entropy of a gaussian packet is plotted. In fact, a very good approximation for the entropy production rate turns out to be;

$$
\dot{\mathcal{H}}=\omega\left(1+\left(\frac{\sigma_{p}^{2}(0)}{\sigma_{c}^{2}}-1\right) \exp (-2 \omega t)\right)^{-1} .
$$

Thus, the transition between reversible area-preserving deformation of the wavepacket and irreversible, diffusive asymptotic regime occurs at the time is;

$$
\tau=\omega^{-1} \log \left(\sigma_{p}(0) / \sigma_{c}\right)
$$


which corresponds to the time for which the spread in momentum becomes comparable with the characteristic dispersion $\sigma_{c}$. The above result should be contrasted with what happens in the case of a stable harmonic oscillator where the entropy growth is slower and depends upon the coupling $\gamma$.

So far, we presented an argument based on the study of the simplest possible exponentially unstable system. The relation with the evolution of chaotic systems is apparent once one realizes that a local analysis around a point in the phase space will reveal the existence of stable and unstable directions, with a "saddle point" structure analogous to an unstable harmonic oscillator ${ }^{13}$. In fact, this is the way one defines local Lyapunov exponents (as the rate at which two neighboring points in phase space tend to separate in time). Thus, the Liouville flow associated with a chaotic Hamiltonian system has, locally, a pattern similar to the one around an hyperbolic point. However, as is well known, the quantum Wigner distribution does not follow the Liouville flow but depart from it after a characteristic time $t_{\chi}$. This crossover time (after which classical and quantum expectation values begin to diverge for chaotic systems) can be found by analyzing the equation for the Wigner function in which the classical Poisson bracket is replaced by the Moyal bracket which contains higher order derivative terms. Assuming one can Taylor expand the potential $V$, the $\mathrm{n}$-th order correction introduced by the Moyal bracket is proportional to;

$$
\mu_{n}=\frac{\hbar^{2 n}}{(2 n+1) ! 2^{2 n}} \partial_{x}^{2 n+1} V \partial_{p}^{2 n+1} W .
$$

Let us define a characteristic lengths associated with the derivatives of the potential as;

$$
\left(\chi_{n}\right)^{2 n} \equiv\left(\partial_{x} V / \partial_{x}^{2 n+1} V\right), n \geq 1,
$$

and introduce a characteristic momentum dispersion as;

$$
\left(\sigma_{p}^{(n)}(t)\right)^{2 n} \propto \partial_{p} W / \partial_{p}^{2 n+1} W .
$$

In terms of these quantities we can easily write the condition for the $\mathrm{n}$-th correction due to the Moyal bracket to be much smaller than the classical terms as;

$$
\hbar \ll \chi_{n} \sigma_{p}(t)
$$

Above, we have assumed that the state has an asymptotic Gaussian form given by (12), so that a single dispersion in momentum suffices to characterize all of its higher derivatives. The condition (18) is, we believe, a key criterion in assuring the correspondence between the quantum and classical dynamics, especially in the context of chaotic quantum systems. It states that, as long as the small scale structure of $W$ is smooth on scales large compared to those on which the force is significantly non-linear (and which are defined by (16)), the Liouville flow and the Moyal evolution will be essentially indistinguishable. In the context of the present 
discussion, this condition for quantum - classical correspondence will be assured whenever the characteristic length;

$$
\ell \equiv \hbar / \sigma_{c}
$$

is small when compared to scales associated with the nonlinearities of the potential.

The cause of the usual "chaotic" problems ${ }^{14}$ with the correspondence between quantum and classical and the reason for their disappearance in presence of effective decoherence can be understood as follows: For a chaotic system, the exponential Liouville flow tends to generate small structure in $W$ exponentially fast. Therefore, one has $\sigma_{p}(t) \propto \sigma_{p}(0) \exp (-\omega t)$. Replacing this, one easily gets the expression for the crossover time;

$$
t_{\chi}=\omega^{-1} \log \left(\sigma_{p}(0) \chi_{n} / \hbar\right)
$$

The condition for the validity of our previous considerations is then that $t_{\chi}$ must be much larger than the time required by the environment to stop the contraction of $\sigma_{p}$. As we pointed out above, this is the time for the transition from the reversible to the irreversible regime $\tau=\omega^{-1} \log \left(\sigma_{p}(0) / \sigma_{c}\right)$. Consequently, the condition for the validity of the "classical" argument is:

$$
\sigma_{c} \chi_{n} \gg \hbar
$$

When this is satisfied, $W$ follows the Liouville flow and the conclusions drawn from the local analysis in phase space can be applied.

We have demonstrated that an open chaotic quantum systems can exhibit, in addition to the very rapid onset of decoherence, a dynamically (nearly) reversible phase of evolution. This period is necessarily followed by an irreversible phase in which the entropy increases linearly at the rate determined by the Lyapunov exponents. By contrast, open quantum systems classical analogs of which do not generate exponentially diverging trajectories will continue to evolve with little entropy production (although possibly with a significant change in dynamics). Thus, quantum dynamics of open systems allows for a qualitative distinction between chaotic and regular motions. It suggests not only a clear distinction between the integrable and chaotic systems, but also shows that increase of entropy in the context of quantum measurement ${ }^{15}$ and the dynamical aspects of the second law are intimately related and can be traced to the same cause: Impossibility of isolating macroscopic systems from their environments.

\section{Existential Interpretation}

Perception of unique events at well-defined instants of time can be naturally accounted for from within the framework of decoherence. All of the arguments against decoherence express dissatisfaction with it as an explanation of our perceptions because it does not force all of the wavefunction of the Universe into a unique state directly corresponding to our experience. Rather, it explicitly assumes that 
the observers are an integral part of the Universe and analyses the measurement-like processes through which perception of the familiar classical reality comes about.

In this setting the observer must be demoted from the position of an all-powerful external experimenter dealing, from without, with one more physical system (the Universe) to a subsystem of that Universe, with all of the limitations arising from such a confinement to within the physical entity he/she is supposed to monitor. Correlation - between the memory of the observer and the outcomes (records) of the past observations - emerges as a central concept. Observers never have enough evidence, enough memory capacity to worry about the state of the Universe as a whole. All they can immediately access is their own records - the state of their own knowledge - and must rely on the fact that this state corresponds (because of the correlations) to certain states of other systems. The veracity of the record is then checked by comparing predictions based on it with the outcomes of the future measurements.

To propose an interpretation - establish the correspondence between quantum formalism and our perceptions, our direct experience - it is crucial to appreciate the double role of the records maintained by the observers: On the one hand, a record is "just a record" - it stores the information acquired by the observer. On the other hand, the record is also the state of a subsystem, defining in part identity of the observer. (Thus, for example, if one were to "copy" an observer, it would necessary to specify also the state of the observers memory!) In this very direct sense "bit is it" and "information is physical" 16 . Conscious observer is (in part) information! The knowledge - records acquired through measurement or communication - become an "identity card" deciding who the observer is: There can be no information without representation.

Modifications of the observers identity as a result of quantum goings on may be drastic (as would be the case for Schrödingers cat) or subtle (as for Wigners friend). Observers may or may not be conscious of them (whatever "being conscious" might mean). Only states which can continue to define both the state of the observer and the state of his/her knowledge for prolonged periods of time, at least as long as the characteristic information - processing timescales of our own nervous system (certainly more than a millisecond, which is in turn many orders of magnitude longer than it takes to decohere) will correspond to perceptions. Information is impossible to separate from the stable existence of records. This requirement of stability and the ultimate sameness of the identity and perceptions define the existential interpretation of quantum mechanics.

The role of diecoherence is to cause the negative selection, and thus define the stable alternatives - states of the observers identity which can persist in spite of their immersion in the environment. The concept of "events" and the "reality" of the states of macroscopic objects can be deduced from within this decoherenceinspired framework: Events happen because the environment defines a set of options (rather small compared to the set of possibilities available in principle in the Hilbert space). Each time the system of interest (or a memory of an apparatus, computer, 
or our own nervous system) is forced into a superposition which violates environment - induced superselection rules, it will decohere on a timescale which is nearly instantaneous when the options are macroscopically distinguishable. This onset of decoherence is the apparent "collapse of the wavepacket." Thereafter, each of the alternatives sprouts a new branch of the Everett's universal state vector. It will evolve on its own, with negligible chances of interference with the other alternatives, but with the correlation of the records with all the relevant states of the measured observables intact.

In nearly all cases effective collapse of the state of a macroscopic object (such as an apparatus) happens irrevocably long before our neurons ever get involved. Thus, the selection of a set of outcomes concludes well in advance of the instant in which "consciousness" can be invoked. Moreover, there is no evidence that any mysterious and essentially quantum ingredient needs to be invoked to explain the operation of our brains.

In spite of the Everett-like framework of this discussion, the picture that emerges in the end is very much in accord with the views of Bohr ${ }^{17}$ : Macroscopic observer will have recording and measuring devices which will behave in a very classical manner. Any measurement will lead to an almost instantaneous reduction of the wavepacket, so that the resulting mixture can be safely regarded as corresponding to just one unknown measurement outcome.

According to the existential interpretation, what observer perceives is not "a wavefunction of the Universe" but its specific branch consistent with all of the records observers state happens to consist of. The freedom to partition the global state vector into any set of branches (present in the original work of Everett) has been significantly constrained by the common sense requirement that the state of the observer (including its memory content) should be able to persist on dynamical timescales, that is for much longer than the decoherence time. Global wavefunction of the Universe - save for the bundle of branches consistent with the identity of the observer, including in particular his/her records - is completely inaccessible. Such observer will perceive events, remember a specific history, and agree about it wiih the others.

We would like to thank Andreas Albrecht, Salman Habib, Jonathan Halliwell, and Raymond Laflamme for discussions and comments. This paper is based in part on an early draft of the manuscript prepared by one of the authors for Physics Today 4 . It is printed here with permission of the American Physical Society.

\section{REFERENCES}

1. A. Einstein, in Albert Einstein, Hedwig und Max Born Briefwechsel, pp. 283-284, Max Born ed. (1969).

2. W. H. Zurek, Physics Today 44, 36 (1991). 
3. W. H. Zurek, "Preferred States, Predictability, Classicality, and the EnvironmentInduced Decoherence," to appear in Physical Origins of Time Asymmetry, J. J. Halliwell, J. Perez-Mercader and W. H. Zurek eds. (Cambridge University Press, Cambridge, in press).

4. W. H. Zurek, Physics Today 46, 81 (1993).

5. W. H. Zurek and J. P. Paz, "Decoherence, chaos and the second law", Los Alamos Preprint LA-UR-93-3514.

6. W. H. Zurek, Phys. Rev. D26, 1862 (1982).

7. A. O. Caldeira and A. J. Leggett, Physica A121, 587 (1983); W. G. Unruh and W. H. Zurek, Phys. Rev. D40, 1071 (1989). B. J. Hu, J. P. Paz and Y. Zhang, Phys. Rev. D45 2843 (1992), ibid, D47 1576 (1993).

8. W. H. Zurek, S. Habib, and J. P. Paz, Phys. Rev. Lett. 70, 1187 (1993).

9. W. H. Zurek, Phys. Rev. D24, 1516 (1981); also, in Experimental Gravitation and the Measurement Theory, P. Meystre and M. O. Scully eds. (Plenum, New York, 1983).

10. R. B. Griffiths, J. Stat. Phys. 36, 219 (1984); R. Omnès, Rev. Mod. Phys 64, 339 (1992), and references therein; M. Gell-Mann and J. B. Hartle, in Complexity, Entropy, and the Physics of Information, W. H. Zurek, ed. (Addison - Wesley, Redwood City, 1990).

11. J. P. Paz and W. H. Zurek, Phys. Rev. D48 (1993) 2728.

12. J. P. Paz, S. Habib and W. H. Zurek, Phys. Rev. D47, 488 (1993).

13. See, e.g. G. Ioos, R. H. G. Hellmann and R. Stora eds., Chaotic Behavior in Deterministic Systems, Les Houches Lectures XXXVI (North Holland, Amsterdam, 1983); R. S. Mc Kay and J. O. Meiss eds., Hamiltonian Dynamical Systems (Hilger, Philadelphia, 1987).

14. M. B. Berry, Proc. Roy. Soc. London, A423, 219 (1989); M. Gutzwiller, Chaos in Classical and Quantum Mechanics (Springer Verlag, New York, 1990); F. Haake, Quantum Signature of Chaos (Springer Verlag, New York, 1990) and references therein.

15. J. von Neumann, Mathematical Foundations of Quantum Mechanics, english translation by R. T. Beyer (Princeton Univ. Press, Princeton, 1955); H. D. Zeh, The Direction of Time, (Springer-Verlag, New York, 1991).

16. J. A. Wheeler, in Complexity, Entropy, and the Physics of Information, W. H. Zurek ed. (Addison- Wesley, Redwood City, 1990); R. Landauer, Physics Today, 44, 23 (1991).

17. N. Bohr, Nature 121580 (1928). 

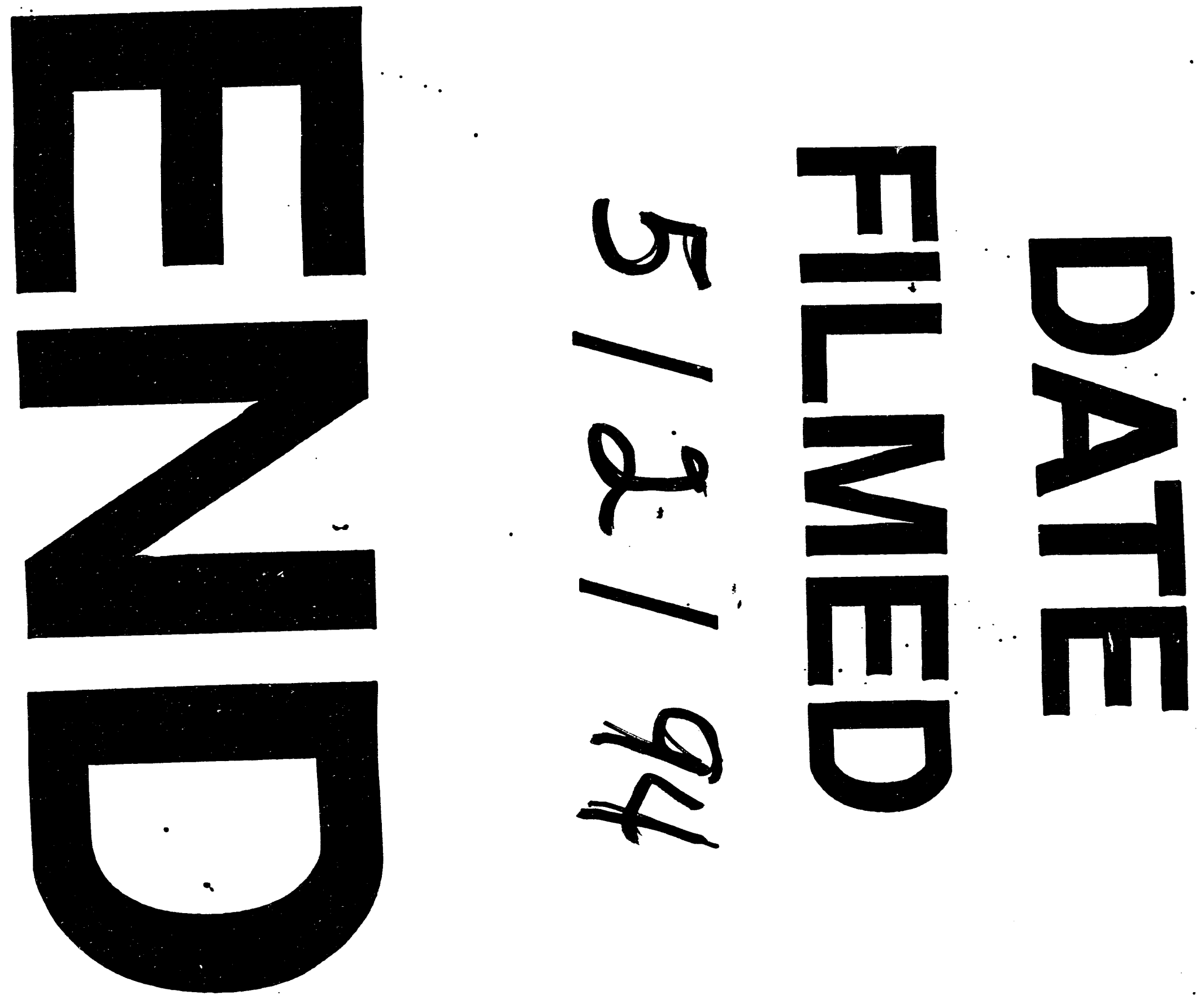
